九州大学学術情報リポジトリ

Kyushu University Institutional Repository

\title{
圧潰前の無症候性大腿骨頭壊死亡症候性大腿骨頭壊 死におけるMRI所見の違い
}

畑中，敬之

https://doi.org/10.15017/2348701

出版情報：Kyushu University，2019，博士（医学），課程博士 バージョン：

権利関係 : 
Research Article

\title{
Differences in magnetic resonance findings between symptomatic and asymptomatic pre-collapse osteonecrosis of the femoral head
}

\author{
Hiroyuki Hatanaka, Goro Motomura*, Satoshi Ikemura, Yusuke Kubo, Takeshi Utsunomiya, \\ Shoji Baba, Koichiro Kawano, Yasuharu Nakashima \\ Department of Orthopaedic Surgery, Graduate School of Medical Sciences, Kyushu University, 3-1-1, Maidashi, Higashi-ku, Fukuoka 812-8582, Japan
}

\section{A R T I C L E I N F O}

\section{Keywords:}

Osteonecrosis

Pre-collapse

Bone marrow edema

\begin{abstract}
A B S T R A C T
Purpose: The purpose of this study was to assess the differences in magnetic resonance (MR) findings between the symptomatic and asymptomatic pre-collapse stage of osteonecrosis of the femoral head (ONFH). Materials and methods: This study reviewed 123 consecutive hips in 91 patients in the pre-collapse stage of ONFH based on plain radiographic findings. These 123 hips were divided into symptomatic and asymptomatic groups according to the pain domain score in the Harris hip score system. Bone marrow edema (BME), synovial fluid effusion, and subchondral fracture were evaluated using MR imaging. Odds ratios (ORs) were calculated between these three parameters and symptoms. The subsequent clinical course after MR examination was also assessed for each hip that could be followed more than 1 year.

Results: Forty-six hips (37.4\%) were categorized as symptomatic and 77 hips (62.6\%) as asymptomatic. The prevalence of BME, synovial fluid effusion, and subchondral fracture were $87.0 \%(40 / 46), 80.4 \%(37 / 46)$, and $34.8 \%(16 / 46)$, respectively, in the symptomatic group, and $0 \%, 28.6 \%(22 / 77)$, and $0 \%$, respectively, in the asymptomatic group, indicating significant differences between the two groups $(\mathrm{p}<0.0001)$. Among these parameters, BME showed the highest OR with regard to the presence or absence of symptoms (BME, 965.8; joint effusion, 10.3; subchondral fracture, 83.9). Due to persistent pain and subsequent collapse, 30 of 35 (85.7\%) symptomatic hips with BME subsequently underwent surgical treatment at a mean interval of 3.47 months after MR examination, while 25 of 66 (37.9\%) asymptomatic hips without BME underwent surgical treatment at a mean interval of 20.7 months after MR examination.

Conclusions: This study demonstrated that symptomatic pre-collapse ONFH diagnosed based on plain radiographic findings could be distinguished from asymptomatic pre-collapse ONFH by the presence of BME on MR imaging, and thus BME may be a sign of occult fracture.
\end{abstract}

\section{Introduction}

The treatment strategy of osteonecrosis of the femoral head (ONFH) is generally decided based on the disease stage, and the success of treatment usually depends on the accuracy of stage classification [1]. Currently, the Ficat and Arlet, Association Research Circulation Osseous (ARCO), and Japanese Investigative Committee (JIC) systems are widely used for stage classification [2-4]. These systems broadly distinguish between the pre- and post-collapse stages based on the shape of the femoral head on plain radiographic findings. However, it is often difficult to assess the pre- or post-collapse stage when there are only minute radiographic changes in the contour of the femoral head. Therefore, the insufficient reproducibility of stage classification has been pointed out [1].

The pre-collapse stage of ONFH is generally considered to be nearly asymptomatic, and the condition is therefore often first diagnosed retrospectively during detailed examination of the contralateral hip in patients with symptoms [5]. Persistent hip pain in ONFH patients is generally thought to be due to subchondral fracture and subsequent femoral head collapse in the post-collapse stage [6], but in many studies

\footnotetext{
Abbreviations: ONFH, osteonecrosis of the femoral head; MR, magnetic resonance; BME, bone marrow edema

* Corresponding author at: Department of Orthopaedic Surgery, Graduate School of Medical Sciences, Kyushu University, 3-1-1, Maidashi, Higashi-ku, Fukuoka 812-8582, Japan.

E-mail addresses: hakkun@ortho.med.kyushu-u.ac.jp (H. Hatanaka), goromoto@ortho.med.kyushu-u.ac.jp (G. Motomura), sikemura@ortho.med.kyushu-u.ac.jp (S. Ikemura), kubokku@ortho.med.kyushu-u.ac.jp (Y. Kubo), takeshiu@ortho.med.kyushu-u.ac.jp (T. Utsunomiya), s-baba@ortho.med.kyushu-u.ac.jp (S. Baba), kawano7@ortho.med.kyushu-u.ac.jp (K. Kawano), yasunaka@ortho.med.kyushu-u.ac.jp (Y. Nakashima).
} 
treatment was performed for symptomatic ONFH despite it being classified as the pre-collapse stage [7-11]. In current classification systems, staging is independent of symptoms, and therefore both painful and asymptomatic hips have been generally classified as the pre-collapse stage if the spherical shape of the femoral head is maintained without apparent subchondral fracture on radiographs. However, grouping symptomatic and asymptomatic pre-collapse stage of ONFH together may complicate evaluations of treatment outcomes.

Magnetic resonance (MR) imaging is a more sensitive examination than radiographs to evaluate the state of the femoral head in ONFH because of its multiplanar imaging capability and the ability to directly image the condition of bone marrow [12]. Bone marrow edema (BME), subchondral fracture, and increased synovial fluid are known to be concomitant and characteristic MR findings in post-collapse ONFH [13-18]. Kim et al. reported that BME was seen most often in stage 3 (post-collapse stage), with high rates of concomitant hip pain, and was not observed in the early stages of disease [14]. Meier et al. also reported a strong correlation between subchondral fracture and BME in the post-collapse stage of ONFH [16]. On the other hand, several authors reported that the appearance of a band pattern without BME was a characteristic initial MR finding in the early stage of ONFH [14,18]. Thus, it has been strongly suggested that MR findings in ONFH differ between hips before and after collapse.

To the best of our knowledge, no studies have investigated which MR differences can distinguish between the presence and absence of hip pain in the radiologically determined pre-collapse stage of ONFH. The purpose of this study was to assess whether there are differences in MR findings between symptomatic and asymptomatic patients with the precollapse stage of ONFH.

\section{Materials and methods}

\subsection{Patient selection}

Ethical approval by the institutional review board was obtained for this retrospective study, and the requirement for informed consent was waived. From January 2010 to March 2016, 386 hips in 250 patients were newly diagnosed with ONFH at our institution. All patients underwent X-ray imaging (anteroposterior and lateral two-dimensional radiographs) and MR imaging, and were diagnosed based on the previously reported criteria for osteonecrosis [3]. Hip diseases with radiological findings similar to ONFH, including transient osteoporosis of the hip and insufficient subchondral fracture, were excluded based on the rigorous MR diagnostic feature of ONFH: a smooth, low-intensity band that is concave to the articular surface and circumscribes all necrotic segments. Contrast-enhanced MR imaging was also performed in cases where the diagnosis of ONFH was difficult [19]. Based on twodimensional (anteroposterior and lateral) radiographs of hip joints at the first diagnosis, the 386 hips were divided into 162 hips with the precollapse stage of ONFH and 224 hips with the post-collapse stage of ONFH. Of the 162 hips with the pre-collapse stage, 39 were excluded because MR examination was performed more than 1 month after the examination of the initial radiographs. Finally, we retrospectively reviewed 123 hips in 91 patients with the pre-collapse stage of ONFH (Fig. 1). The patients with the pre-collapse stage included 51 males (73 hips) and 40 females (50 hips) with a mean age of 43.1 years (14-76 years). Their body mass index (BMI) was $23.1 \mathrm{~kg} / \mathrm{m}^{2}(15.2-33.9 \mathrm{~kg}$ / $\mathrm{m}^{2}$ ). Regarding the factors associated with ONFH, 51 patients (71 hips) had a history of steroid administration, 26 patients (34 hips) had a history of alcohol abuse, 5 patients (6 hips) had both histories (steroid and alcohol use), and 9 patients (12 hips) had no relevant history (idiopathic). Regarding the localization of the necrotic lesion according to the JIC classification, 16 hips were classified as Type A (the necrotic lesion occupies the medial one-third or less of the weight-bearing portion), 21 hips as Type B (the medial two-thirds or less of the weightbearing portion), 54 hips as Type C1 (more than two-thirds of the weight-bearing portion but not extending to the acetabular rim), and 32 hips as Type C2 (more than two-thirds of the weight-bearing portion and extending to the acetabular rim) [3].

\subsection{Evaluation of symptomatic and asymptomatic hips}

According to the pain domain score in the Harris hip score [20] at the time of initial medical examination, 123 hips with the radiological pre-collapse stage of ONFH were divided into symptomatic or asymptomatic hips. Patients with 30 or fewer points (mild pain, no effect on common activities, rare moderate pain with unusual activity) were classified as symptomatic, and patients with 40 or 44 points were classified as asymptomatic.

\subsection{Definition of pre-collapse on radiographs}

The pre-collapse stage of ONFH was defined when both of the following two conditions were met (Fig. 2). First, the contour of the femoral head maintained a spherical shape. In detail, a spherical shape was defined when no point on the circumference of a perfect circle centered on the femoral head was farther than $1 \mathrm{~mm}$ from the contour of the actual articular surface, on both anteroposterior and lateral radiographs. The second condition was that no crescent sign was seen within the femoral head. The radiological evaluation was conducted using the Image $\mathrm{J}$ software program (National Institutes of Health, Bethesda, MD, USA), which can measure length on radiographs in 0.1mm units. Definition of pre-collapse was made blindly by two authors (H.H and Y.K) who had extensive experience in the diagnosis of ONFH. If both authors made different judgments, the case was considered to represent the post-collapse stage of ONFH.

\subsection{MR imaging evaluations}

Patients with the pre-collapse stage of ONFH underwent MR examination at our institution using a 1.5-T or 3-T MR system (Achieva; Philips Medical Systems, Best, The Netherlands) within a month after the first radiographic examination. The mean duration between radiograph and MR examination was 12.5 days (0-31 days). Coronal and oblique axial planes on T1-weighted images (TR/TE: 400-600/ 8-19 ms) and fat-suppressed T2-weighted images (TR/TE: 3000-4000/ $80-108 \mathrm{~ms}$ ) were obtained in the supine position with $5-\mathrm{mm}$ slice thickness, 1-mm inter-slice gap, and field of view of $360 \times 360 \mathrm{~mm}$. Three parameters (Fig. 3), namely BME, synovial fluid effusion, and subchondral fracture, were evaluated on all slices. Positive BME was defined when a diffuse, low-signal intensity area on T1-weighted images with a corresponding high-signal intensity on fat-suppressed T2weighted images was seen around the femoral head and neck beyond the necrotic lesion $[13,14,21,22]$. Synovial joint effusion was considered to be present when it was observed around the femoral neck on fat-suppressed T2-weighted images [17]. Subchondral fracture was defined as positive when an irregular, low-intensity band running parallel to the subchondral bone plate on T1-weighted images was seen along with corresponding bands of low or high signal intensity on fatsuppressed T2-weighted images away from the boundary of the necrotic lesion $[14,16]$. All MR imaging evaluations were performed by consensus of two authors (H.H and Y.K) who had extensive experience in the examination of $\mathrm{ONFH}$.

\subsection{Evaluation of clinical course after MR imaging}

Using both follow-up radiographs and medical records, the clinical course after MR examination was assessed for each hip that could be followed up for more than 1 year. This evaluation included the presence or absence of apparent femoral head collapse and surgical treatments. 


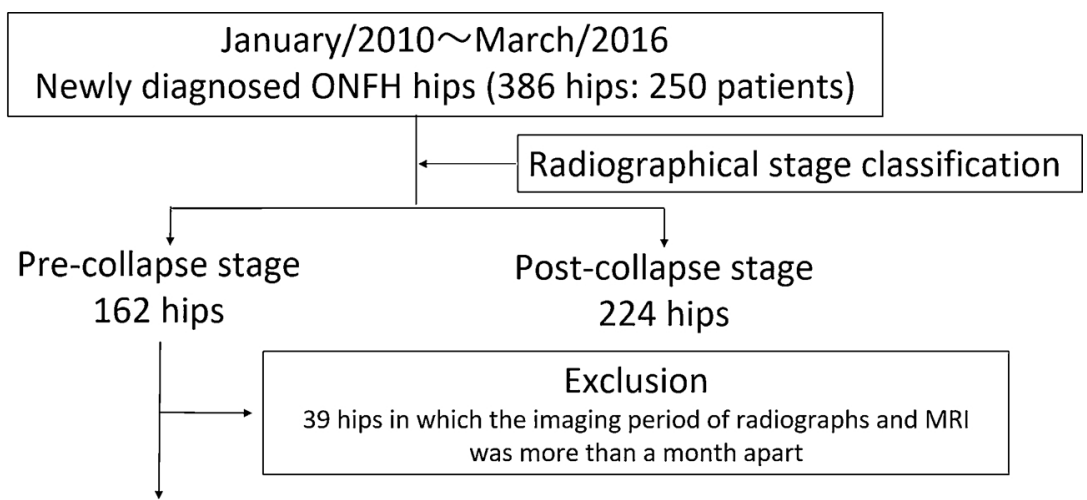

Our subjects: 123 hips of 91 patients

Fig. 1. Flowchart demonstrating selection of patients with the pre-collapse stage of osteonecrosis of the femoral head.

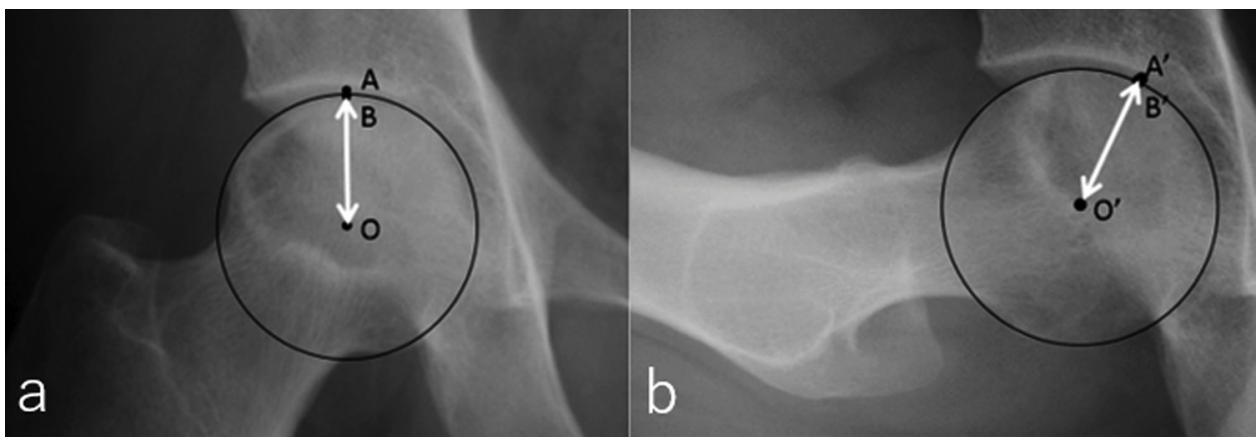

Fig. 2. The pre-collapse stage of osteonecrosis of the femoral head was defined when the distances between OA (O'A') and OB (O'B') were both less than $1 \mathrm{~mm}$, and no subchondral fracture line was seen. A or A': the intersection point of a circle concentric to the femoral head with the line passing through the center of the femoral head (O or O'). B or B': the intersection point of the articular surface with the line passing through the center of the femoral head.

\subsection{Statistical analysis}

Statistical analyses were carried out using Fisher's exact probability test to compare the ratio of three parameters, namely BME, synovial fluid effusion, and subchondral fracture, between symptomatic and asymptomatic hips. Odds ratios (ORs) were also calculated between these three parameters and symptoms. Student's $t$-test was used to determine the association between MR findings and the period from MR examination until surgery due to persistent pain and subsequent collapse. A Kaplan-Meier analysis was performed and survival curves were created with the log-rank test. A value of $p<0.05$ was considered to indicate significance. These statistical analyses were performed using JMP statistical analysis software (version 13; SAS Institute, Cary, NC, USA).

\section{Results}

Forty-six hips (37.4\%) were categorized as symptomatic and 77 hips $(62.6 \%)$ as asymptomatic. On MR, BME was observed in $40(32.5 \%)$ hips, synovial fluid effusion was observed in 59 (48.0\%) hips, and subchondral fracture was observed in 16 (13.0\%) hips.

As shown in Table 1, the prevalence of BME, synovial fluid effusion, and subchondral fracture were $87.0 \%(40 / 46), 80.4 \%(37 / 46)$, and $34.8 \%$ (16/46), respectively, in the symptomatic group (Fig. 4), and $0 \%$ (0/77), $28.6 \%(22 / 77)$, and $0 \%(0 / 77)$, respectively, in the asymptomatic group (Fig. 5). The prevalence of these three parameters were significantly higher in the symptomatic group than in the asymptomatic group ( $\mathrm{p}<0.0001$ for all three parameters). Among these parameters, BME showed the highest OR with regard to the presence or absence of hip pain (BME: 965.8, joint effusion: 10.3, subchondral fracture: 83.9 ) (Table 1). There was a significant relationship between these MRI parameters and the localization of the necrotic lesion (Table 2).
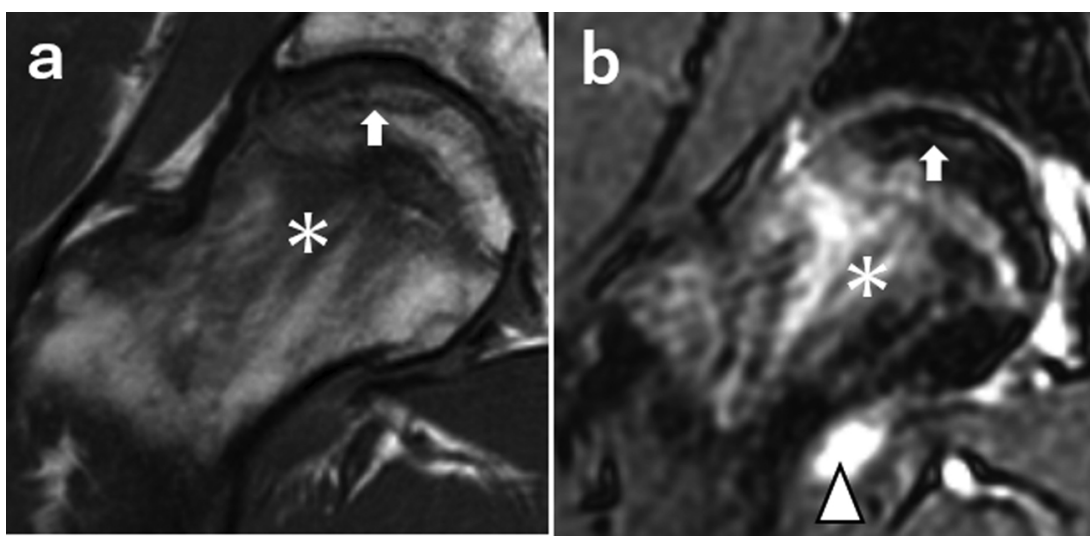

Fig. 3. Coronal T1-weighted MR image (a) and fat-suppressed T2-weighted image (b). BME manifested as a diffuse, lowsignal intensity area on T1-weighted images with a corresponding high-signal intensity on a fat-suppressed T2weighted image around the femoral head and neck beyond the necrotic lesion and extending to the intertrochanteric lesion (asterisk). Synovial joint effusion (arrowhead) was defined as positive when it surrounded the femoral neck on a fat-suppressed T2-weighted image (b). Subchondral fracture (white arrow) was defined as positive when an irregular, low-intensity band running parallel to the subchondral bone plate on a T1-weighted image (a) and corresponding bands of low or high signal intensity on a fat-suppressed T2-weighted image (b) were seen beyond the boundary of the necrotic lesion. 
Table 1

Relationship between hip pain and MR parameters.

\begin{tabular}{|c|c|c|c|c|c|}
\hline Parameters of MR findings & & $\begin{array}{l}\text { Symptomatic } \\
(\mathrm{n}=46)\end{array}$ & $\begin{array}{l}\text { Asymptomatic } \\
(\mathrm{n}=77)\end{array}$ & $\mathrm{p}$ value & Odds ratio $(95 \% \mathrm{CI})$ \\
\hline \multirow[t]{2}{*}{ Bone marrow edema (BME) } & Presence & $40(87.0 \%)$ & $0(0 \%)$ & $<0.0001^{*}$ & $965.8^{\text {k* }}$ (84.8 to 10015 ) \\
\hline & Absence & $6(13.0 \%)$ & $77(100 \%)$ & & \\
\hline \multirow[t]{2}{*}{ Synovial fluid effusion } & Presence & $37(80.4 \%)$ & $22(28.6 \%)$ & $<0.0001^{*}$ & 10.3 \\
\hline & Absence & $9(19.6 \%)$ & $55(71.4 \%)$ & & (4.26 to 24.8 ) \\
\hline \multirow[t]{2}{*}{ Subchondral fracture } & Presence & $16(34.8 \%)$ & $0(0 \%)$ & $<0.0001^{*}$ & $83.9^{\text {*ktk }}$ \\
\hline & Absence & $30(65.2 \%)$ & $77(100 \%)$ & & ( 8.17 to 838 ) \\
\hline
\end{tabular}

* p values less than 0.05 indicate a significant difference.

$* *$ Cell data of 0 were corrected by adding 0.5 to all values of the cell.

Of the 123 total hips, 106 were followed up for more than 1 year. Fifty-six hips underwent surgery due to persistent pain with subsequent collapse. Forty-two hips were observed conservatively without radiological collapse, and eight were observed conservatively despite confirmation of a minimal collapse. Due to persistent pain and subsequent collapse, 30 of 35 (85.7\%) symptomatic hips with BME subsequently underwent surgical treatment at a mean interval of 3.47 months (SD 4.02) after MR examination, while 25 of 66 (37.9\%) asymptomatic hips without BME underwent surgical treatment at a mean interval of 20.7 months (SD 13.9) after MR examination. Of the remaining five symptomatic hips without BME, one hip underwent surgical treatment at 1 month after MR examination. Kaplan-Meier survivorship analysis using the log-rank test showed a significant difference in the survival rate between the BME-positive and -negative groups (Fig. 6).

\section{Discussion}

Recently, surgical treatments including core decompression with or without stem cell implantation have been increasingly used in the precollapse stage of ONFH to prevent femoral head collapse [7-11]. The "pre-collapse stage" in the currently used ONFH classification systems commonly applies to both symptomatic and asymptomatic hips without any findings of femoral head collapse or subchondral fracture on plain radiographs. The current radiograph-based classification is a very convenient method and has been used as a reference when considering the treatment strategy of ONFH, however in the current study, $37.4 \%$ symptomatic cases and $62.6 \%$ asymptomatic cases were classified as the same pre-collapse stage despite showing the same radiographical findings. We therefore believe that it would be preferable to define any findings that can more accurately classify these symptomatic and asymptomatic pre-collapse stage hips.

In the current study, BME was the MR finding that best distinguished symptomatic from asymptomatic pre-collapse stage of ONFH. In addition, pre-collapse hips with BME generally required surgery for subsequent collapse earlier than those without BME. Although it remains controversial whether or not BME itself can cause hip pain, some studies have proposed that the elevated intramedullary pressure produced by BME is related to hip pain in ONFH [23,24], while other studies have shown that BME on MR findings is more often seen when painful femoral head collapse has already developed $[13,14,18]$. Therefore, it seems reasonable to assume that BME on MR occurs secondarily to subchondral fracture and fracture-associated hip pain in radiographically defined pre-collapse ONFH.

The current study also showed that synovial fluid effusion was a common finding on MR imaging in the symptomatic pre-collapse stage of ONFH. On the other hand, some asymptomatic hip joints also demonstrated effusion but none exhibited BME. Considering the fact that joint effusion is seen in some normal asymptomatic hips [17,23,25], we suppose that synovial fluid effusion is not always associated with pain in the pre-collapse stage of ONFH.

This study has several limitations. First, this was a retrospective study. As a result, our analyses were based on routinely performed examinations, namely plain radiographs and MR imaging. Regarding the detection of subchondral fracture, MR imaging is inferior to computed tomography due to the ambiguity of the fracture line resulting from diffuse BME [16]. We therefore consider that the prevalence of subchondral fracture in symptomatic pre-collapse ONFH was underrepresented in the current study. A prospective analysis should be performed to clarify the association between symptomatic pre-collapse ONFH and subchondral fracture. Second, the presence or absence of hip

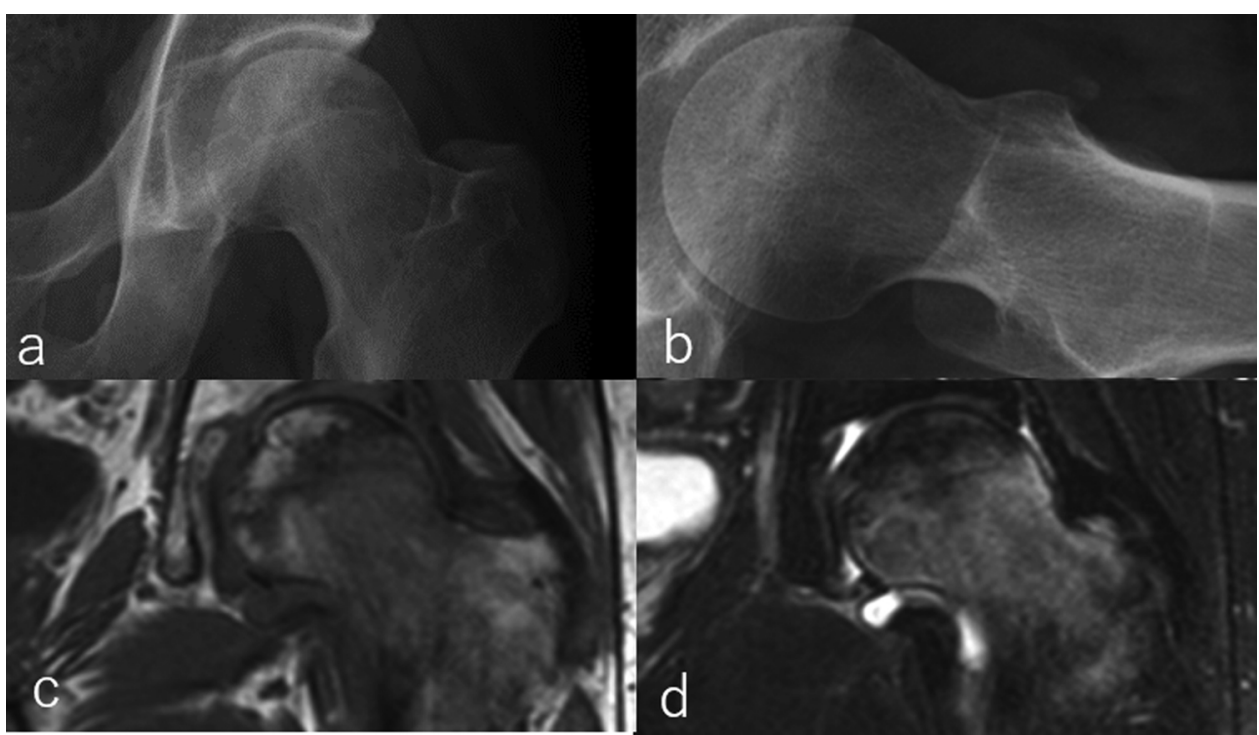

Fig. 4. Radiographic and MR findings in symptomatic patients with the pre-collapse stage of osteonecrosis of the femoral head. On anterior (a) and lateral radiographs (b), the contour of the femoral head maintains a spherical shape without apparent subchondral fracture or collapse. MR findings show bone marrow edema around the femoral head and neck beyond the necrotic lesion, extending to the intertrochanteric lesion on both a coronal T1-weighted image (c) and a fat-suppressed T2-weighted image (d). 


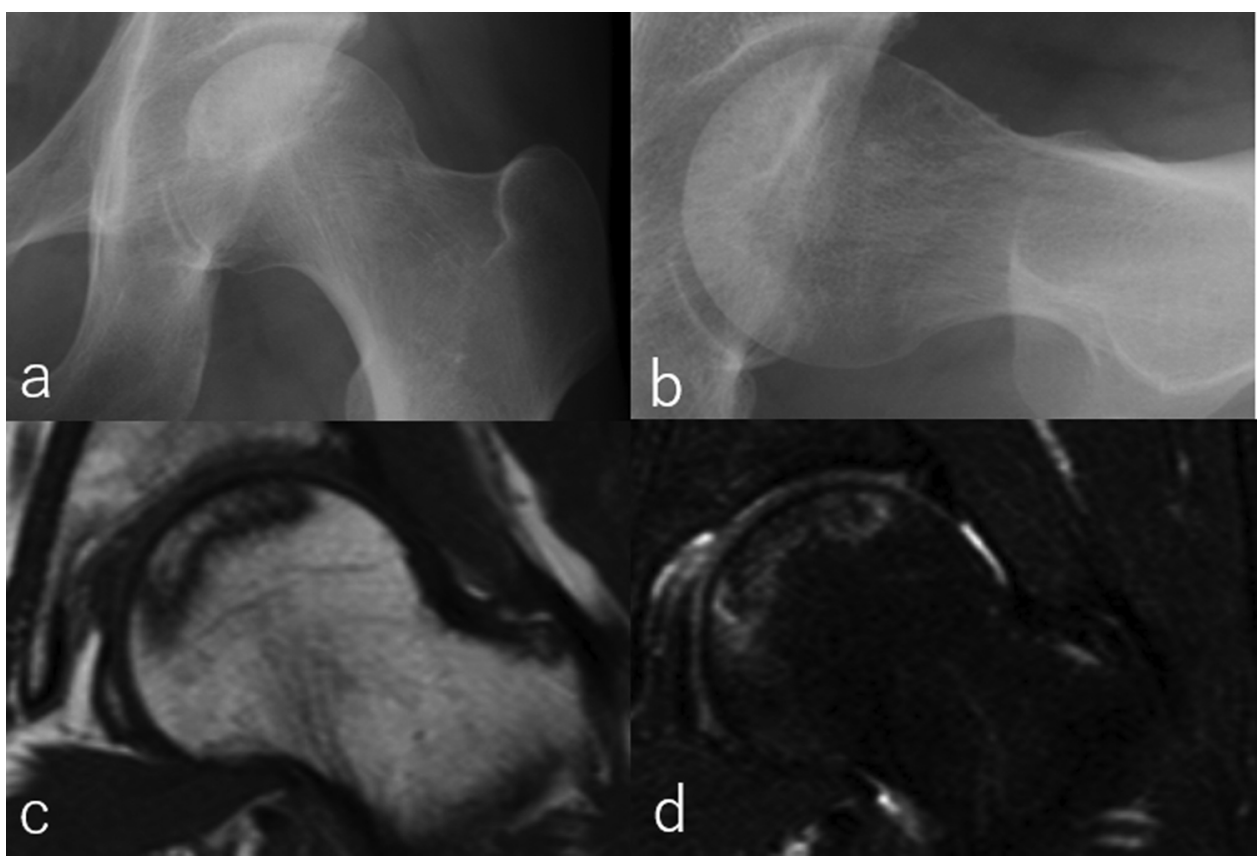

Fig. 5. Radiographic and MR findings in asymptomatic patients with the pre-collapse stage of osteonecrosis of the femoral head. The contour of the femoral head is spherical on anterior (a) and lateral (b) radiographs. Bone marrow edema is not seen on either a coronal T1-weighted image (c) or a fat-suppressed T2weighted image (d).

Table 2

Relationship between MR parameters and localization of the necrotic lesion.

\begin{tabular}{|c|c|c|c|c|c|c|}
\hline \multicolumn{2}{|l|}{ MR parameters } & \multicolumn{4}{|c|}{$\begin{array}{l}\text { Localization of the necrotic lesion } \\
\text { (type) }\end{array}$} & \multirow[t]{2}{*}{$\mathrm{p}$ value } \\
\hline & & $\begin{array}{l}\text { A } \\
(n=16)\end{array}$ & $\begin{array}{l}\mathrm{B} \\
(\mathrm{n}=21)\end{array}$ & $\begin{array}{l}\mathrm{C} 1 \\
(\mathrm{n}=54)\end{array}$ & $\begin{array}{l}\mathrm{C} 2 \\
(\mathrm{n}=32)\end{array}$ & \\
\hline \multirow{2}{*}{$\begin{array}{c}\text { Bone marrow } \\
\text { edema } \\
\text { (BME) }\end{array}$} & Presence & 0 & 4 & 21 & 15 & \multirow[t]{2}{*}{$0.001^{*}$} \\
\hline & Absence & 16 & 17 & 33 & 17 & \\
\hline \multirow{2}{*}{$\begin{array}{c}\text { Synovial fluid } \\
\text { effusion }\end{array}$} & Presence & 2 & 6 & 31 & 20 & \multirow[t]{2}{*}{$0.001^{*}$} \\
\hline & Absence & 14 & 15 & 23 & 12 & \\
\hline \multirow{2}{*}{$\begin{array}{l}\text { Subchondral } \\
\text { fracture }\end{array}$} & Presence & 0 & 0 & 11 & 5 & \multirow[t]{2}{*}{$0.03^{*}$} \\
\hline & Absence & 16 & 21 & 43 & 27 & \\
\hline
\end{tabular}

* p values less than 0.05 indicate a significant difference.

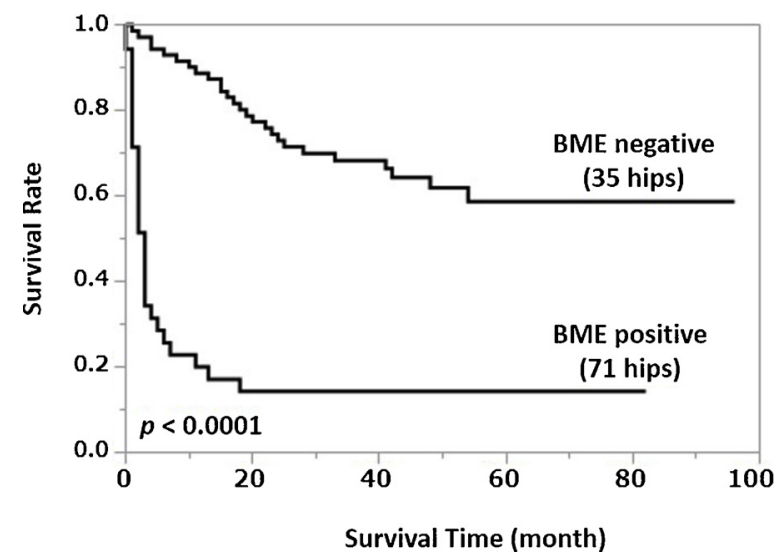

Fig. 6. Kaplan-Meier survival curve shows the survival rate. The endpoint is the time when surgery was performed.

pain was assessed using the Harris hip score, which took into account only medical records data. However, MR findings were clearly different between symptomatic and asymptomatic groups that were defined based on the Harris hip score. Although not ideal, we think that pain assessment using the Harris hip score is clinically useful as a guide for symptomatic pre-collapse ONFH. Third, this study was performed at a single institution. Therefore, there may have been some bias, for instance involving patient selection and staging methods. A multicenter study would be preferable to minimize this bias and increase the generalizability of the results.

In conclusion, this study demonstrated that symptomatic pre-collapse stage of ONFH diagnosed by plain radiography could be distinguished from asymptomatic pre-collapse stage of ONFH by the presence of BME on MR imaging, and that BME may be a sign of occult fracture.

\section{Conflict of interest}

No benefits of any kind have been received or will be received from commercial parties related directly or indirectly to the subject of this article.

\section{Acknowledgments}

This work was supported in part by a grant-in-aid in Scientific Research (16K10906) from the Japan Society for the Promotion of Science. We thank Junji Kishimoto, a statistician from the Digital Medicine Initiative Kyushu University, for his advice on the statistical analysis.

\section{References}

[1] A.Y. Plakseychuk, M. Shah, S.E. Varitimidis, H.E. Rubash, D. Sotereanos, Classification of osteonecrosis of the femoral head: reliability, reproducibility, and prognostic value, Clin. Orthop. Relat. Res. 386 (2001) 34-41.

[2] R.P. Ficat, Idiopathic bone necrosis of the femoral head. Early diagnosis and treatment, J. Bone Joint Surg. Br. 67 (1985) 3-9.

[3] N. Sugano, T. Atsumi, K. Ohzono, T. Kubo, T. Hotokebuchi, K. Takaoka, The 2001 revised criteria for diagnosis, classification, and staging of idiopathic osteonecrosis of the femoral head, J. Orthop. Sci. (7) (2002) 601-605.

[4] J. Gardeniers, ARCO committee on terminology and staging, ARCO News Lett. (1993).

[5] M.A. Mont, M.G. Zywiel, D.R. Marker, M.S. McGrath, R.E. Delanois, The natural history of untreated asymptomatic osteonecrosis of the femoral head, J. Bone Joint Surg. Am. 92 (2010) 2165-2170.

[6] K. Ohzono, M. Saito, K. Takaoka, K. Ono, T. Nishina, T. Kadowaki, Natural history of nontraumatic avascular necrosis of the femoral head, J. Bone Joint Surg. Br. 73 (1) (1991) 68-72.

[7] E. Powell, W. Lanzer, M. Mankey, Core decompression for early osteonecrosis of the hip in high risk patients, Clin. Orthop. Relat. Res. 335 (1997) 181-189.

[8] D. Markel, C. Miskovsky, T. Sculco, P. Pellicci, E. Salvati, Core decompression for 
osteonecrosis of the femoral head, Clin. Orthop. Relat. Res. 323 (1996) 226-233.

[9] B. Mazières, F. Marin, P. Chiron, L. Moulinier, J.M. Amigues, M. Laroche, et al., Influence of the volume of osteonecrosis on the outcome of core decompression of the femoral head, Ann. Rheum. Dis. 56 (1997) 747-750.

[10] T. Floerkemeier, F. Thorey, D. Daentzer, M. Lerch, P. Klages, H. Windhagen, et al., Clinical and radiological outcome of the treatment of osteonecrosis of the femoral head using the osteonecrosis intervention implant, Int. Orthop. 35 (2011) 489-495.

[11] V. Gangji, V. De Maertelaer, J.-P. Hauzeur, Autologous bone marrow cell implantation in the treatment of non-traumatic osteonecrosis of the femoral head: five year follow-up of a prospective controlled study, Bone 49 (2011) 1005-1009.

[12] A.H. Zibis, A.H. Karantanas, N.T. Roidis, M.E. Hantes, P. Argiri, T. Moraitis, et al., The role of MR imaging in staging femoral head osteonecrosis, Eur. J. Radiol. 63 (2007) 3-9.

[13] T. Sakai, N. Sugano, T. Nishii, K. Haraguchi, T. Ochi, K. Ohzono, MR findings of necrotic lesions and the extralesional area of osteonecrosis of the femoral head, Skeletal Radiol. 29 (2000) 133-141.

[14] Y.M. Kim, H.C. Oh, H.J. Kim, Y. Kim, H. Oh, H. Kim, et al., The pattern of bone marrow oedema on MRI in osteonecrosis of the femoral head, J. Bone Joint Surg. Br. Ed. 82 (2000) 837-841.

[15] K. Stevens, C. Tao, N. Salem, J. Vandevenne, C. Chenq, G. Neumann, et al., Subchondral fractures in osteonecrosis of the femoral head: comparison of radiography, CT, and MR imaging, AJR Am. J. Roentgenol. 180 (2003) 363-368.

[16] R. Meier, T.M. Kraus, C. Schaeffeler, S. Torka, A.M. Schlitter, K. Specht, et al., Bone marrow oedema on MR imaging indicates ARCO stage 3 disease in patients with AVN of the femoral head, Eur. Radiol. 24 (2014) 2271-2278.

[17] D.G. Mitchell, V. Rao, M. Dalinka, C.E. Spritzer, W.B. Gefter, L. Axel, et al., MRI of joint fluid in the normal and ischemic hip, Am. J. Roentgenol. 146 (1986) 1215-1218.

[18] T. Kubo, S. Yamazoe, N. Sugano, M. Fujioka, S. Naruse, N. Yoshimura, et al., Initial MRI findings of non-traumatic osteonecrosis of the femoral head in renal allograft recipients, Magn. Reson. Imaging 15 (1997) 1017-1023.

[19] S. Ikemura, T. Yamamoto, G. Motomura, Y. Nakashima, T. Mawatari, Y. Iwamoto, MRI evaluation of collapsed femoral heads in patients 60 years old or older: differentiation of subchondral insufficiency fracture from osteonecrosis of the femoral head, Am. J. Roentgenol. 195 (2010) W63-68.

[20] W.H. Harris, Traumatic arthritis of the hip after dislocation and acetabular fractures: treatment by mold arthroplasty, J. Bone Joint Surg. Am. 51 (1969) 737-755.

[21] S. Iida, Y. Harada, K. Shimizu, M. Sakamoto, S. Ikenoue, T. Akita, et al., Correlation Between Bone Marrow Edema and Collapse of the Femoral Head in Steroid-Induced Osteonecrosis, Am. J. Roentgenol. 174 (2000) 735-743.

[22] H. Ito, T. Matsuno, A. Minami, Relationship between bone marrow edema and development of symptoms in patients with osteonecrosis of the femoral head, Am. J. Roentgenol. 186 (2006) 1761-1770.

[23] K.H. Koo, I.O. Ahn, R. Kim, H.R. Song, S.T. Jeong, J.B. Na, et al., Bone marrow edema and associated pain in early stage osteonecrosis of the femoral head: prospective study with serial MR images, Radiology 213 (1999) 715-722.

[24] M.D. Murphey, K.L. Foreman, M.K. Klassen-Fischer, M.G. Fox, E.M. Chung, M.J. Kransdorf, From the radiologic pathology archives imaging of osteonecrosis: radiologic-pathologic correlation, RadioGraphics 34 (2014) 1003-1028.

[25] G.-S. Huang, W.P. Chan, Y.-C. Chang, C.-Y. Chang, C.-Y. Chen, J.S. Yu, MR imaging of bone marrow edema and joint effusion in patients with osteonecrosis of the femoral head: relationship to pain, AJR Am. J. Roentgenol. 181 (2003) 545-549. 